

Universidad Nacional de Ingeniería COPYRIGHT @ (UNI). TODOS LOS DERECHOS RESERVADOS

http://revistas.uni.edu.ni/index.php/Nexo

https://doi.org/10.5377/nexo.v34i04.12637

Vol. 34, No. 04, pp. 1129-1139/Septiembre 2021

(c) (i) $\odot$

\title{
Modelo viable de empresa creativa para pequeñas y medianas empresas artesanales
}

\section{Viable model of creative company for small and medium art crafts enterprises}

\author{
Citlalli Macías Barreto * y Mario Aguilar Fernández \\ Instituto Politécnico Nacional. Unidad Profesional Interdisciplinaria de Ingeniería y Ciencias Sociales y \\ Administrativas, Sección de Estudios de Posgrado e Investigación. Ciudad de México, México \\ *cmaciasb1800@alumno.ipn.mx
}

(recibido/received: 16-mayo-2021; aceptado/accepted: 08-junio-2021)

\begin{abstract}
RESUMEN
Las empresas artesanales indígenas en México enfrentan dificultades para coordinar y sistematizar sus procesos de diseño y hacer frente a las artesanías de importación. Tras la revisión crítica de las metodologías de diseño de producto, con base en el enfoque del Modelo de Sistemas Viables y aplicando la Metodología de Sistemas Suaves mediante entrevistas semiestructuradas e investigación acción participativa; se propone un Modelo Viable de Empresa Creativa, para mejorar la capacidad de adaptación de estas empresas con énfasis en los sistemas identificados como relevantes para la gestión del diseño. Los resultados son un marco de referencia y diagnóstico para pequeñas y medianas empresas, que deseen mejorar su viabilidad sistémica incorporando la gestión del diseño a sus procesos operativos.
\end{abstract}

Palabras claves: Gestión de diseño; Modelo de empresa; Diagnóstico organizacional; Industrias Creativas.

\begin{abstract}
Indigenous artisan companies in Mexico, face difficulties in coordinating and systematizing their design processes and dealing with imported handicrafts. After critical review of product design methodologies, based on the Viable Systems Model approach and applying the Soft Systems Methodology through semistructured interviews and participatory action research; A Viable Model for a Creative Enterprise is proposed to improve the adaptability of these companies with emphasis on the systems identified as relevant for design management. The results are a reference and diagnostic framework for small and medium enterprises that wish to improve their systemic viability by incorporating design management into their operational processes.
\end{abstract}

Keywords: Design management; Company model; Organizational diagnosis; Creative Industries. 


\section{INTRODUCCIÓN}

La contribución al Producto Interno Bruto de las empresas de la economía creativa es cada vez más reconocida por los gobiernos del mundo, lo que se refleja en sus planes de desarrollo económicos (Grobar, 2019; United Nations Conference on Trade and Development [UNCTAD], 2018). Esto representa una oportunidad para que las Pequeñas y Medianas Empresas (PYME) de este sector se acerquen a sus objetivos organizacionales. La categoría más importante de la economía creativa en términos de exportación es la de bienes creativos, que incluye: Diseño, Artes Visuales, Nuevos medios de comunicación, Artesanía, Publicación, Medios Audiovisuales y Artes escénicas. Para el año 2015, el subsector más importante de estas industrias fue el de productos de diseño, representando el $62 \%$ del comercio mundial de esta categoría; en contraste, las Artesanías representan el 7\%, solo por encima de Medios Audiovisuales y Artes escénicas (UNCTAD, 2018, p. 22).

Al igual que los productos incluidos en la categoría de diseño, los productos artesanales abarcan una amplia diversidad de técnicas y bienes de uso y consumo; sin embargo, los productos artesanales se encuentran excluidos de esa categoría en un intento por separar los procesos culturales y cognitivos, de las expresiones materiales y sus medios de producción, ya sean manuales o industriales. Paradójicamente, son cada vez más los diseñadores profesionales que aprovechan procesos artesanales para la producción de bienes creativos capaces de competir en un mercado global, dado el atractivo contenido cultural emanado de sus contextos de producción, por ejemplo: (Ceccarelli, 2019; Kalkreuter, 2020; Kulick, 2017; Lavin, 2019; Marques et al., 2019; Milgram, 2020). Por lo anterior, es posible asumir que el éxito de los productos diseñados se relaciona más con la capacidad de gestión del diseño, que por el producto mismo o su nivel de industrialización.

Toda artífice es por naturaleza producción humana, y en consecuencia, todo producto es de diseño, ya sea deliberado o arbitrario, profesional o intuitivo; e inevitablemente intrínseco a su contexto cultural y de producción material. A pesar de que el mercado mundial de artesanías creció durante el período 20032015 con una tasa promedio anual de 4,42\%, México participa solo con el $0.6 \%$ a una tasa promedio anual de $-3.09 \%$; muy por debajo del dominio de China, que cuenta con un $48.7 \%$ del mercado y tasa de crecimiento de $12.9 \%$, seguida de Turquía e India con el $7.7 \%$ y $4.5 \%$ del mercado, con tasas de crecimiento promedio de $13.58 \%$ y $6.31 \%$ respectivamente (UNCTAD, 2018, p. 26). En contraste, los bienes de diseño mexicano representaron el principal grupo de exportación de bienes creativos en 2014 con \$3.5 millones, tres veces por encima del segundo grupo en relevancia; mientras que las artesanías representaron solo \$248 millones para el mismo año, cifra inferior a la reportada para 2005 (UNCTAD, 2018, p. 304).

De lo anterior se infiere que en México existe conocimiento en diseño susceptible de ser incorporado en empresas artesanales para favorecer su producción, y aspirar a equilibrar la balanza de exportaciones dado que, con base en el mismo reporte, se importa tres veces más artesanía de la que se exporta, lo que representa una amenaza para los productores nacionales. Con la finalidad de contribuir al fortalecimiento de la PYME artesanal en México, y dada su importancia para las regiones habitadas por los pueblos originarios, esta investigación se centró en proponer un Modelo Viable de Empresa Creativa concebido para mejorar la capacidad de adaptación de las PYME dedicadas a la producción artesanal, ante el entorno cada vez más cambiante y globalizado.

\section{METODOLOGÍA}

Se empleó la metodología de Intervención Total de Sistemas (Jackson, 2002) para la planificación, diseño, evaluación y resolución de problemas empleando el pensamiento de sistemas críticos; a partir del cual se seleccionó como marco teórico el Modelo de Sistemas Viables (Beer, 1985) para el diagnóstico organizacional; y la Metodología de Sistemas Suaves (Checkland y Poulter, 2010), para la recolección y 
procesamiento de los datos. Dado el enfoque cualitativo y de investigación acción participativa, los métodos e instrumentos seleccionados fueron: observación participante, grupos de enfoque y entrevistas semiestructuradas y estructuradas. Para la construcción de los datos se consideraron solo empresas artesanales rurales e indígenas, ubicadas en pueblos originarios de antigua historia y cultura compartida. Se inició con la revisión crítica de los modelos metodológicos para el diseño de productos más influyentes de los últimos 50 años (Vilchis-Esquivel, 2014), con la finalidad de recuperar los fundamentos, elementos y momentos del diseño profesional hasta lograr la saturación teórica.

Con la finalidad de identificar los modelos de empresa existentes en la región, y su experiencia en procesos de diseño ya utilizados, se realizaron 10 entrevistas a profundidad con preguntas semiestructuradas a tomadores de decisiones en seis empresas artesanales, seleccionadas por muestreo aleatorio por conveniencia y efecto de bola de nieve debido a que no se cuenta con registros públicos y confiables para calcular un muestreo técnico estratificado. El rigor metodológico se cumplió por el método de panel de expertos, triangulación y dependencia interna de los datos. Con base en el análisis de los datos y de la revisión crítica de los procesos de diseño, se propuso un Modelo Tentativo de Empresa Creativa siguiendo el Modelo de Sistemas Viables (MSV), empleado posteriormente como guía para la discusión ordenada durante cuatro semanas de investigación acción participativa en una séptima empresa artesanal, con la finalidad de mejorarlo y detallarlo siguiendo la Metodología de Sistemas Suaves tras lo cual, se obtuvo el Modelo por Consenso de Empresa Creativa.

\section{RESULTADOS}

En este apartado se presentan y describen los resultados obtenidos tras aplicar la estrategia metodológica descrita en apartados anteriores. Se inicia presentando los resultados de la revisión crítica de los recursos de diseño identificados como relevantes, obtenidos por saturación teórica, de este modo se identifican cinco fases indispensables en toda metodología de diseño de producto, estrechamente relacionadas y cuyo orden responderá al razonamiento lógico más adecuado para cada caso, por lo que aquí se presentan como un modelo metodológico general para diseño de productos (Figura 1). Identificadas las fases indispensables en toda metodología de diseño de producto y tras la construcción y procesamiento de los datos con base en la MSS (Checkland y Poulter, 2010), fue posible definir la situación problemática de investigación como es mostrado a continuación.

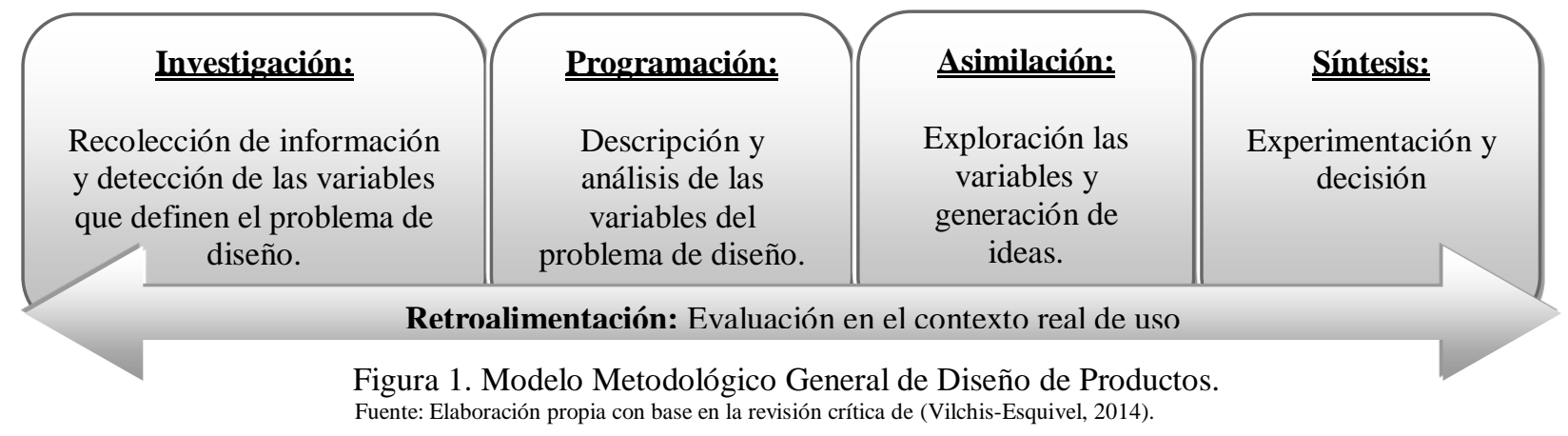

Las empresas artesanales indígenas, tienden a adoptar una estructura organizacional flexible en red, constituida por células de trabajo que suelen ser unidades económicas familiares distribuidas geográficamente en la comunidad. Por tal motivo, pocas veces comparten el espacio físico simultáneamente para la toma de decisiones y el trabajo productivo. Ante esta distribución, las PYME artesanales enfrentan dificultades para coordinar el trabajo, así como sistematizar y socializar los procesos de diseño de producto. A partir de los resultados de las dos etapas previamente descritas y con base en Beer (1985), se propone el Modelo Tentativo de Empresa Creativa (Figura 2), donde se destacan como relevantes, el Sistema cuatro (S4.), y el Sistema tres (S3.). Lo anterior con base en la situación 
problemática detectada y en alineación con el MSV, ya que es en estos dos subsistemas donde se llevan a cabo los procesos de diseño, pensados para ser ejecutados en un sistema viable. Una vez en el contexto de la empresa participante, el Modelo Tentativo de Empresa Creativa de la Figura 2, fue empleado como guía para la discusión ordenada llevada a cabo, mediante grupos de enfoque y observación participante con base en Checkland y Poulter (2010). Mediante consenso de las distintas visiones del mundo recuperadas de todos los actores con propósito involucrados, fue posible desarrollar cada uno de los cinco subsistemas del modelo, para adecuarlo al contexto estudiado.

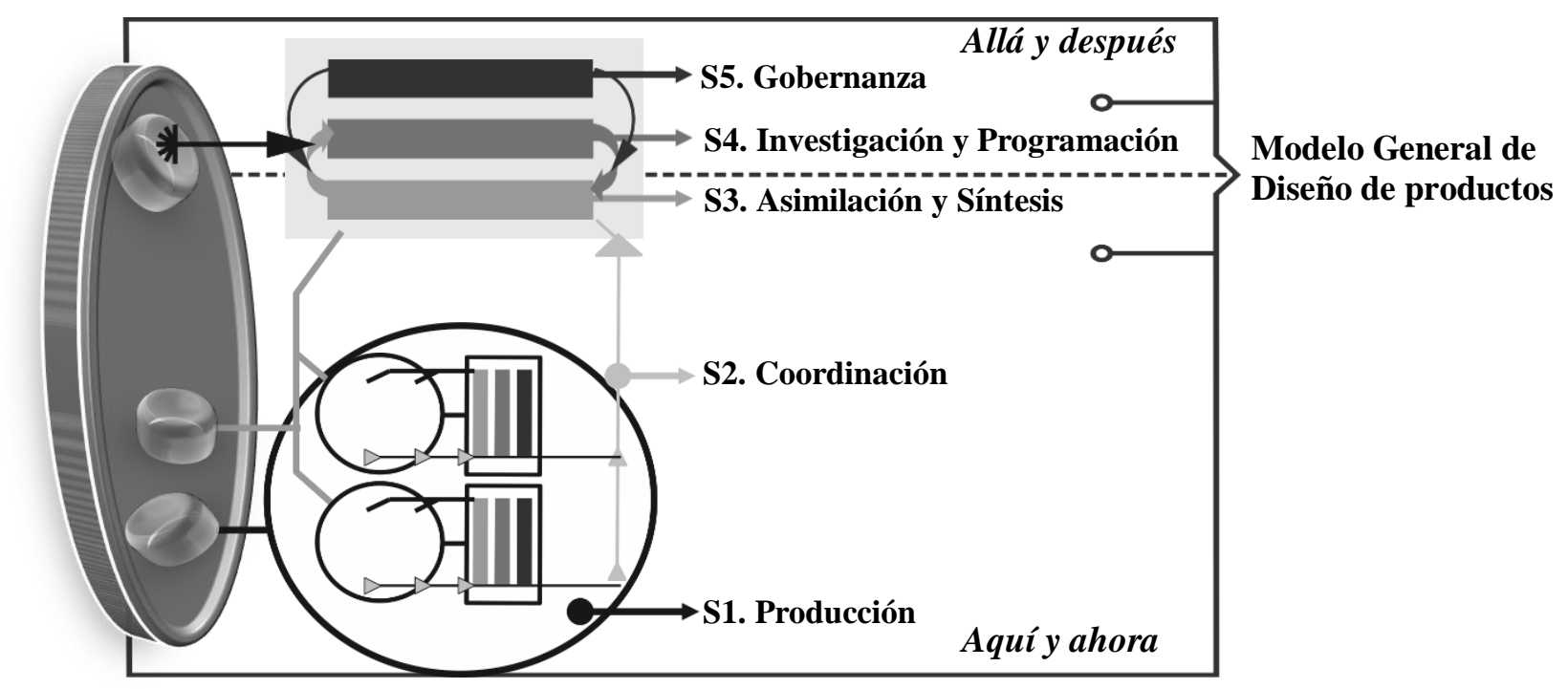

Figura 2. Modelo Tentativo de Empresa Creativa. Fuente: Elaboración propia con base en (Beer, 1985).

Con base en lo anterior en la Figura 3 se presenta, el Modelo Viable de Empresa Creativa para Empresas Artesanales integrando el Modelo General de Diseño de Productos (Figura 1), y del Modelo Tentativo (Figura 2). Como puede observarse, cada subsistema se encuentra interrelacionado con el resto de la empresa cumpliendo así el principio de interdependencia entre las partes, que genera un estado de búsqueda constante de equilibrio mediante la auto regulación simultáneamente en diferentes niveles. Dada la complejidad del modelo y para facilitar la descripción de cada subsistema del Modelo Viable de Empresa Creativa propuesto en la Figura 3, a continuación, se describen las operaciones, entradas, salidas, mecanismos y controles necesarios para cada caso.

Descripción del S1. Producción. Este es el subsistema responsable de los procesos de transformación de materias primas. Para este nivel se propone una disposición en células productivas con horarios y agendas flexibles, lo que responde a las condiciones de la estructura en red identificada en empresas artesanales. De este modo, la producción se distribuye en pequeños grupos que realizan de principio a fin el proceso de producción de una fracción del volumen total, en congruencia con los procesos artesanales. El proceso inicia con el Reparto que implica la división de insumos provenientes de Almacén de Materiales, acompañados de instrucciones y material de apoyo para la producción por medio de RTRO CON, y concluye con las salidas o bienes procesados por cada Célula en almacén de Producto Terminado (Figura 4). 


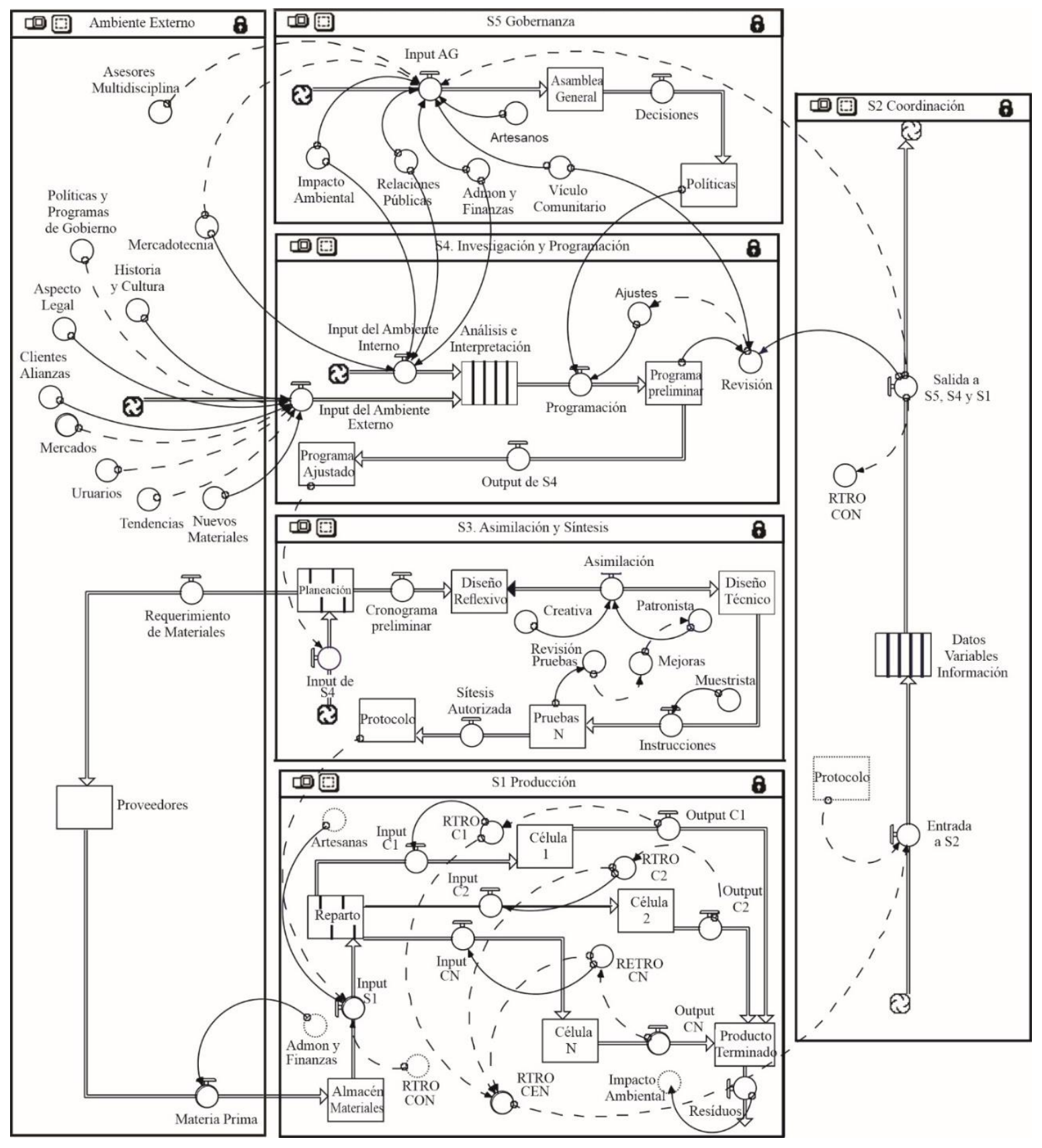

Figura 3. Modelo Viable de Empresa Creativa para Empresas Artesanales.

Fuente: Elaboración propia a partir de los datos con apoyo del software iThink, versión 8.0 para Windows, con base en la Figura 2.

Descripción del S2. Coordinación. Este es el subsistema responsable de recabar información y datos provenientes de todos los subsistemas con la finalidad de alinear todas las acciones conforme a lo planeado. De este modo se valora la información por parte del subsistema responsable para responder a cada evento oportunamente, lo que mantiene el flujo del trabajo y permite suministrar respuestas de contingencia, supervisión y planeación estratégica. Dado que cada Célula en el S1. Producción realiza todas las operaciones de producción, el subsistema $S 2$. Coordinación introduce las instrucciones a cada una mediante RTRO CON al momento del Reparto de materiales. Asimismo, cada Célula de trabajo contempla un bucle de 
retroalimentación $R T R O C 1, R T R O C 2, R T R O C N \ldots$, que reporta datos, eventos o información (tales como: merma o desperdicios, tiempos de los procesos de producción y demoras, tiempos de ejecución de prototipos, consumo y calidad de materiales, puntos críticos de producción, etc.) al S2. Coordinación para su recolección por medio de RTRO CEN, con la finalidad de canalizarlos al subsistema correspondiente para su procesamiento. El proceso de coordinación inicia con la entrada al sistema de un Protocolo que contiene todas las instrucciones y variables de diseño (serie de pautas de decisión) que prefiguran la producción de cada proyecto, y el formato de cronograma preliminar para ser negociado con cada Célula de trabajo. La operación de coordinación se lleva a cabo durante todo el tiempo que el proyecto esté vigente y finaliza con la comunicación de los datos recolectados a cada una de las partes correspondientes (Figura 5).

Descripción del S3. Asimilación y Síntesis. Este es el subsistema responsable de abordar los aspectos tanto creativos como técnicos del producto. Es un proceso reflexivo acotado por los procesos de producción de la empresa y su finalidad es generar valor para el entorno. El proceso inicia cuando el Programa Ajustado y los datos preliminares del cronograma, entran a este subsistema para ser interpretados y asimilados, tomando en cuenta las políticas formuladas en el $S 5$. Gobernanza y los datos recolectados por $S 2$. Coordinación. Del proceso de experimentación y mejora de pruebas, se obtiene un prototipo afinado que sintetiza todas las variables del problema plasmadas en un Protocolo de diseño para cada proyecto con los parámetros, puntos críticos, maquinaria y equipo necesarios para la producción, así como las órdenes de compra de los materiales necesarios para suministrar al S1. Producción (Figura 6).

Descripción del S4. Investigación y Programación. Este subsistema es el responsable de llevar a cabo las operaciones de inteligencia mediante la investigación y comprensión del problema de diseño y su programación, acorde al presente pero con la mirada puesta en el futuro definido por el subsistema S5. Gobernanza. El proceso inicia con entrada de información desde múltiples fuentes del entorno de la empresa para ser analizada y procesada desde los diferentes enfoques departamentos o áreas de la empresa y/o asesores externos. Para su autorización, dicha información se plasma en un Programa preliminar para replantearse con base en la información recabada por el S2. Coordinación mediante un enfoque decolonial y horizontal. El proceso termina con la autorización de un Programa Ajustado a los criterios organizacionales, donde se describen las variables y especificidades del problema de diseño e incluye, un formato de cronograma preliminar para la planeación del trabajo y compras (Figura 7).

Descripción del S5. Gobernanza. Este subsistema es el responsable de explorar los posibles estados de la organización, y las actividades de inteligencia enfocadas a asegurar el equilibrio entre las decisiones tomadas por los subsistemas S4. Investigación y Programación, y S3. Asimilación y Síntesis. Se encuentra conformada por la Asamblea General, la cual es el máximo órgano de dirección y de la toma de decisiones de la empresa artesanal. Se celebra periódicamente e inicia estableciendo el orden del día por acuerdo, se recibe información analizada por el S4. Investigación y Programación y recopilada por el S2. Coordinación, así como asesoría y consejo externo multidisciplinario según se requiera. Posteriormente se discute cada punto mediante el uso libre de la palabra para llegar a acuerdos o votaciones sobre las decisiones que dan rumbo, sentido y propósito al largo plazo, reflejadas en las directrices y políticas que guían el comportamiento organizacional y perfilan su identidad empresarial (Figura 8). 
Entradas:

Protocolo y cronograma acordado desde RTRO CON.

Materiales desde Almacén.

Mecanismos:

Artesanos organizados en células de trabajo

Maquinaria y herramienta en función de la técnica.
Control:

Variables del problema de diseño.

Tiempos de entrega.

Figura 4. Esquema del Subsistema 1. Producción.

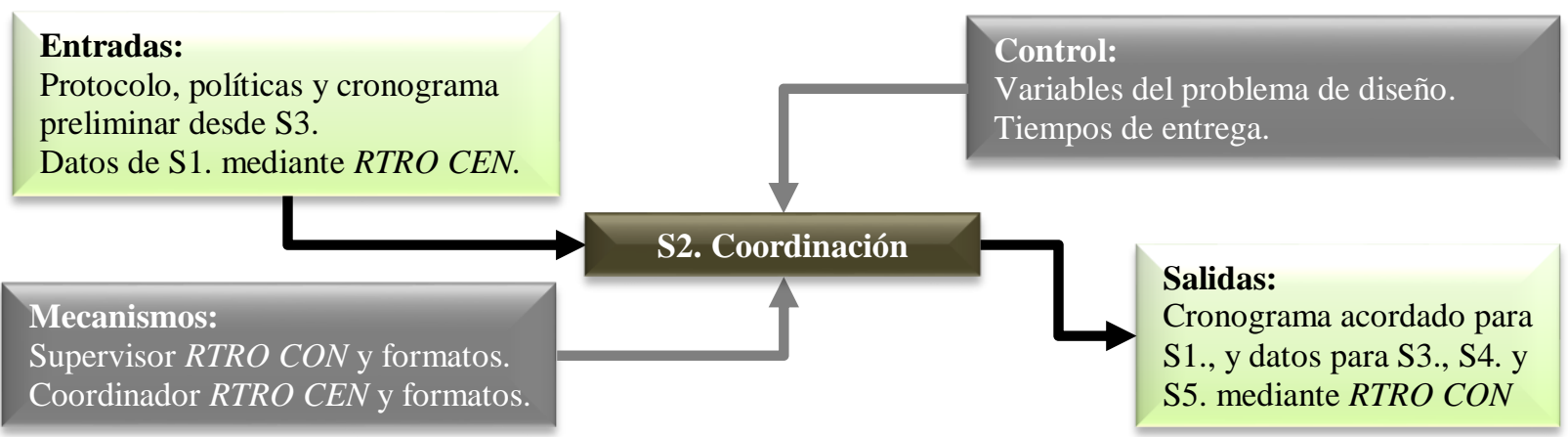

Figura 5. Esquema del Subsistema 2. Coordinación.

Salidas:

Producto terminado y material residual. Datos mediante RTRO CEN.

\section{Entradas:}

Programa ajustado desde S4.

Cronograma preliminar.
Control:

4.

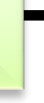



Parámetros de decisión y variables del problema de diseño. Políticas y lineamientos desde S5.

S3. Asimilación y Síntesis

\section{Mecanismos:}

Diseñador creativo - técnico, instrumentos para el dibujo y molduras.

Muestrista, maquinaria y herramienta en

función de la técnica.



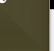

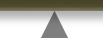

Figura 6. Esquema del Subsistema 3. Asimilación y Síntesis.

Entradas:

Información desde el ambiente interno y múltiples fuentes del entorno externo de la empresa.
Mecanismos:

Distintas visiones del problema.

Acceso a fuentes de información.

Cámara, impresora, notas, audios, etc.

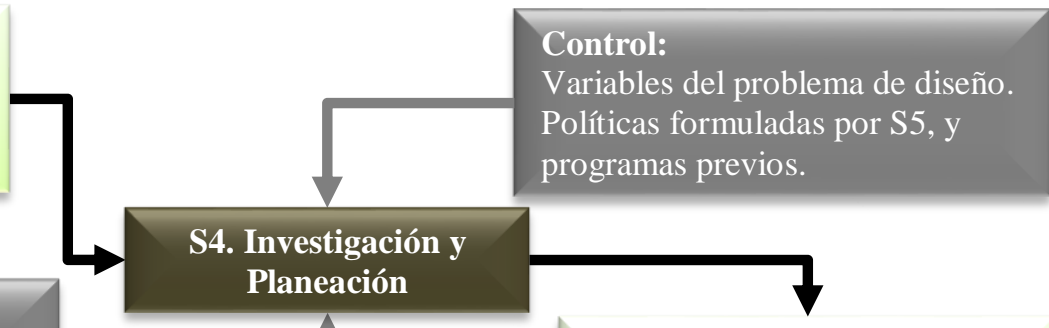

Salidas:

Programa de diseño ajustado y cronograma preliminar.

Figura 7. Esquema del Subsistema 4. Investigación y Programación. 




Figura 8. Esquema del Subsistema 5. Gobernanza.

\section{DISCUSIÓN}

Resulta difícil comparar los resultados obtenidos en investigaciones previas debido al uso impreciso del término modelo de empresa, pues éste frecuentemente se confunde con el modelo de negocio o giro de la empresa (Caban-Piaskowska, 2019); el cual responde a la mezcla estratégica de elementos comerciales mediante el cual, la empresa capitaliza el valor percibido por los consumidores. Por lo anterior se subraya que en este documento, el modelo viable de empresa se entiende como la estructura operativa y de ingeniería de procesos mediante la cual una empresa genera valor para su entorno y es capaz de adaptarse a los cambios de éste; por lo que se diferencia de los estudios sobre modelos comerciales de negocios (Johnson et al., 2008).

Los resultados obtenidos proporcionan un marco para identificar las fortalezas y debilidades sistémicas de PYMES artesanales en términos de la gestión de sus procesos de diseño de producto, experiencia similar a la de (Brocklesby y Cummings, 1996) en su intervención y consultoría en Telecom con la MSV, a pesar de las diferencias en la escala de las empresas estudiadas. Los resultados no pueden comparase con los obtenidos del Modelo de Capacidad de Diseño (Storvang et.al., 2014), el cual se enfoca en identifica el nivel de asimilación de la práctica del diseño desde cinco dimensiones distintas de la empresa (percepción del diseño, en qué procesos es importante, participación de los usuarios, motor de innovación y origen de las capacidades de diseño), cada una con niveles propios que van desde la inexistencia de la dimensión, hasta el punto más alto de compromiso con la práctica bajo criterios no definidos.

Por su parte, en la literatura sobre el tema, existe el consenso de que la Empresa Creativa (EC) es aquella que genera productos o servicios de arte comerciales, tanto tangibles como intangibles, e incluso se han identificado algunas características que, paradójicamente no son exclusivas de éstas, sino que las comparte con empresas que emplean la gestión de diseño ${ }^{1}$ en su modelo de empresa (Caban-Piaskowska, 2019). Para efectos del presente documento se recuperan solo algunas que se relacionan con los resultados obtenidos para su discusión: Se concuerda en que la estructura de la EC debe caracterizarse por la formación de equipos multidisciplinarios orientados a trabajar por proyecto, pero se difiere en que es la cooperación lo que les permite reaccionar rápidamente ante cambios imprevistos (Caban-Piaskowska, 2019). En esta propuesta, la capacidad de respuesta en tiempo y forma depende de el correcto funcionamiento del S2. Coordinación, quien tiene la función de mantener todas las partes integradas y alineadas lo que le permite capacidad de respuesta oportuna, independientemente de la naturaleza de los lazos entre los miembros de la empresa.

${ }^{1}$ Con base en Caban-Piaskowska (2019, p. 164), "la gestión del diseño es una gestión eficiente de las personas, proyectos, procesos y procedimientos en el diseño de productos, servicios, medio ambiente cotidianos. $\mathrm{y}$ experiencias". 
Se concuerda en que la EC hace énfasis en la adecuación de los productos a las especificidades de los clientes (Johnson et al., 2008); sin embargo, se difiere en que éstas no se preocupan por la estandarización o la reducción de los costos (Caban-Piaskowska, 2019), pues este pensamiento resulta contradictorio en términos de gestión de diseño que, como se mencionó antes, es inherente a la EC En cambio se estima que justamente este punto amerita atención en las EC, particularmente en las PYMES artesanales, pues al carecer de sistematización de procesos y estándares, se incurre en desaprovechamiento de recursos de todo tipo. Se concuerda con Johnson et al. (2008) en que la empresa que se centra en fortalecer las conexiones entre sus procesos logra diferenciarse de las que no lo hacen, en especial cuando se trata de procesos clave que generan valor, como es el caso de los de diseño. La perspectiva de investigación se concuerda con (Brocklesby y Cummings, 1996), quienes reconocen en el MSV, un enfoque evolutivo y mesurado con los cambios en las organizaciones, pues mediante el diagnóstico se fortalecen las similitudes de la empresa con relación al modelo y que se deben mantener; por otro lado, el diagnóstico evidencia las debilidades o diferencias en relación al modelo ideal sobre las cuales debe centrarse el cambio, pues la mayoría de las empresas se encuentran fuera de la esfera de modelos disruptivos.

Por su parte, se coincide con Topaloğlu y Er (2017), en que la importancia de la gestión del diseño irá en aumento, dada su capacidad de detonar mejoras en múltiples dimensiones de la empresa, como es la mejora de su desempeño, bienestar social y ambiental, reflejado en el Modelo Viable de Empresa Creativa propuesto, pues la evolución del campo de la gestión del diseño revela que también es aplicable al contexto organizacional (Topaloğlu y Er, 2017). En este sentido, no basta con identificar características de la EC y proponer modelos de empresa deseables pues, como menciona Topaloğlu y Er (2017), para que las empresas desarrollen capacidades de diseño y su gestión, son necesarias herramientas y metodologías que las acerquen al modelo planteado como el ideal a alcanzar, y en este sentido aún resta mucho por hacer.

\section{CONCLUSIONES}

Tras la discusión de los resultados se concluye que, el Modelo Viable de Empresa Creativa aquí presentado, es útil para diagnosticar el estado de los sistemas que intervienen en la gestión del diseño en empresas artesanales, con la finalidad de identificar los cambios deseables y culturalmente posibles que la empresa deberá impulsar; y de este modo, acercarse al estado organizacional deseable representado en el modelo por consenso. Es importante señalar que para acercarse al modelo, además de formular recomendaciones con base en éste, es necesario emplear una metodología que permita asimilar los cambios deseados mediante la repetición de procedimientos para su sistematización. El enfoque del MSV permite una perspectiva estructurada y ágil para el diagnóstico de fenómenos complejos, como lo son las empresas sin importar su tamaño; sin embargo, aún son pocas las investigaciones empíricas y teóricas para la comparación de resultados análogamente, por lo que se alienta su utilización en futuras investigaciones. En este sentido, se recomienda emplear el modelo aquí propuesto para el análisis en las PYME de manufactura que deseen mejorar su viabilidad dado que, el diseño se encuentra implícito en toda actividad humana, independientemente de su nivel de profesionalización o uso intuitivo.

Por lo anterior, la presente investigación se aleja de los intentos de separar, el intelecto y la cultura, de la producción material humana manifiesta sensorialmente, pues éstos no pueden ser disociados. Dada la tendencia global de los mercados, serán cada vez más las empresas las que incorporen la gestión del diseño a su estructura operativa, por lo que parece estéril clasificar como un sector particular las industrias creativas; y aún más, distinguirlas con base en lo disruptivo de su modelo comercial, pues lo nuevo solo es nuevo una vez; en cambio, la capacidad creadora se desarrolla e incrementa sobre la base de lo anterior, pues su naturaleza es evolutiva y continua. El Modelo Viable de Empresa Creativa aquí propuesto difiere de los modelos comerciales de negocio, pues en este documento se modela el estado viable que una empresa puede adoptar, vista como un conjunto complejo de procesos vitales de toda PYME productiva. Si bien no se descalifica la relevancia de definir la fórmula comercial del modelo de negocios, desde la 
perspectiva de la presente investigación que aborda el enfoque de sistemas y la gestión del diseño, en este documento la E.C. se define por su capacidad de adaptarse y mantener comunicación consciente con su entorno, empleando para ello la gestión del diseño.

\section{REFERENCIAS}

Basole, A. (2015). Authenticity, Innovation, and the Geographical indication in an artisanal industry: the case of the Banarasi sari. Journal of World Intellectual Property, 18(3-4), 127-149. https://doi.org/10.1111/jwip.12035

Beer, S. (1985). Diagnosing the system for organizations. Oxford, Great Britain: Oxford University Press.

Brocklesby, J., y Cummings, S. (1996). Designing a viable organization structure. Long Range Planning, 29(1), 49-57. https://doi.org/10.1016/0024-6301(95)00065-8

Caban-Piaskowska, K. (2019). Business model of a creative company and design management. Problemy Zarzadzania, 2/2019(82), 155-169. https://doi.org/10.7172/1644-9584.82.8

Ceccarelli, N. (2019). Neo-Local design. Looking at 'our local contexts' as potential resources. Design Journal, 22(sup1), 931-946. https://doi.org/10.1080/14606925.2019.1595409

Checkland, P., y Poulter, J. (2010). Soft system methology método radical para integrar actividades organizativas (1 ra.). Barcelona, España: BookPrint * Hospitalet de Llobregat.

Grobar, L. M. (2019). Policies to promote employment and preserve cultural heritage in the handicraft sector. International Journal of Cultural Policy, 25(4), 515-527. https://doi.org/10.1080/10286632.2017.1330887

Jackson, M. C. (2002). Systems approaches to management. New York, USA: Kluwer Academic Publisher.

Johnson, M. W., Christensen, C. M., y Kagermann, H. (2008). Reinventing your business model. Harvard Business Review, 86(12), 57-68. Recuperado de http://syv.pt/login/upload/userfiles/file/Reinventing Your business model HBR.pdf\#page $=57$

Kalkreuter, B. (2020). Anyone's heritage? indian fashion design's relationships with craft between local guardianship and valorization of global fashion. Fashion Practice, 12(2), 264-287. https://doi.org/10.1080/17569370.2020.1769361

Kulick, G. (2017). Adding plus value to development aid projects through design strategy: experiences from Pakistan. The Design Journal, 20(sup1), S411-S423. https://doi.org/10.1080/14606925.2017.1352939

Lavin, M. C. (2019). Craft and design partnerships in the chilean context. A critical perspective. Design Journal, 22(sup1), 967-979. https://doi.org/10.1080/14606925.2019.1595411

Marques, C. S., Santos, G., Ratten, V., y Barros, A. B. (2019). Innovation as a booster of rural artisan entrepreneurship: a case study of black pottery. International Journal of Entrepreneurial Behaviour and Research, 25(4), 753-772. https://doi.org/10.1108/IJEBR-02-2018-0104

Milgram, B. L. (2020). Fashioning frontiers in artisanal trade: social entrepreneurship and textile 
production in the Philippine Cordillera. South East Asia Research, 28(4), 1-19. https://doi.org/10.1080/0967828X.2020.1834336

Storvang, P., Jensen, S., y Christensen, P. R. (2014). Innovation through design: a framework for design capacity in a danish context. Design Management Journal, 9(1), 9-22. https://doi.org/10.1111/dmj.12006

Topaloğlu, F., y Er, Ö. (2017). Discussing a new direction for design management through a new design

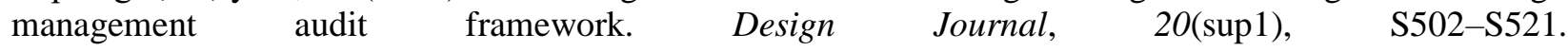
https://doi.org/10.1080/14606925.2017.1353000

United Nations Conference on Trade and Development. (2018). Crative economy outlook trends in international trade in creative industries 2002-2015. Recuperado de https://unctad.org/en/pages/PublicationWebflyer.aspx?publicationid=2328

Vilchis-Esquivel, L. del C. (2014). Metodología del diseño Fundamentos Teóricos (4ta ed.). Ciudad de México, México: D.R. Editorial Designio.

\section{SEMBLANZA DE LOS AUTORES}

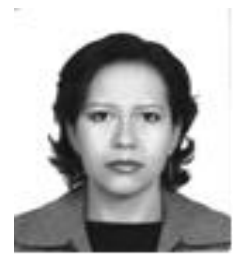

Citlalli Macías Barreto: Obtuvo el grado de Licenciada en Diseño de Indumentaria y Textiles en la Universidad Autónoma de Aguascalientes, México. Actualmente realiza estudios de posgrado en el programa de la Maestría en Ciencias en Estudios Interdisciplinarios para Pequeñas y Medianas Empresas en la Sección de Estudios de Posgrado e Investigación de la Unidad Profesional Interdisciplinaria de Ingeniería y Ciencias Sociales y Administrativas del Instituto Politécnico Nacional, México. Es miembro del Seminario de Investigación Microinnovación desde noviembre de 2019. Coautora por primera vez, no cuenta con publicaciones previas, su actual línea de investigación se vincula al crecimiento y desarrollo de la micro, pequeña y mediana empresa artesanal en México, con la finalidad de fortalecerlas y coadyuvar a la consolidación de sus derechos de autonomía dentro del Estado pluricultural mexicano, empleando para ello, enfoques sistémicos y metodologías decoloniales.

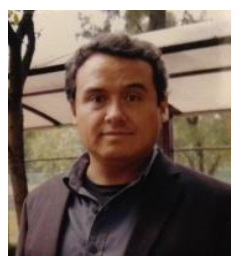

Mario Aguilar Fernández. Integrante del núcleo académico básico de la Maestría en Ingeniería Industrial, y de la Maestría en Ciencias en Estudios Interdisciplinarios para Pequeñas y Medianas Empresas, de la UPIICSA del IPN, México. Es Ingeniero Industrial, con maestría en Ingeniería Industrial por la UPIICSA del IPN, Candidato a Doctor en Ingeniería Industrial en Planificación Estratégica de la Tecnología por el CADIT, de la Universidad Anáhuac del Norte, y, estudiante del Doctorado en Ingeniería de Sistemas en el IPN-ESIME-SEPI, con especialidad en Sistemas Complejos. Es autor de artículos científicos (JCR, CONACyT y SCOPUS), libros, capítulos, y ha participado en congresos nacionales e internacionales. Integrante de la Red de Desarrollo Económico del IPN. 